\title{
Predicting microbiologically defined infection in febrile neutropenic episodes in children: global individual participant data multivariable meta-analysis
}

Robert S Phillips, Lillian Sung, Roland A Ammann, Richard D Riley, Elio Castagnola, Gabrielle M Haeusler, Robert Klaassen, Wim JE Tissing, Thomas Lehrnbecher, Julia Chisholm, Hana Hakim, Neil Ranasinghe, Marianne Paesmans, lan M Hann and Lesley A Stewart on behalf of the PICNICC Collaboration

Correction to: British Journal of Cancer (2016) 114, 623-630. doi:10.1038/bjc.2016.28; published online 8 March 2016

Upon publication of the above paper in the British Journal of Cancer, the authors identified an error in the spelling of one of their names. The publishers would like to apologise for this mistake. Roland A Ammann appears correctly in the author list above.

In addition, the corresponding author was incorrectly designated Professor Robert S Phillips. The correct designation should have been Dr Robert S Phillips. 